\title{
A NONINVERTIBLE UNWEIGHTED BILATERAL SHIFT
}

\author{
RALPH GELLAR AND ROBERT SILBER
}

ABSTRACT. A noninvertible unweighted bilateral shift is constructed in a Nakano $l^{p_{i}}$ space.

To Hilbert space operator theorists our title may seem paradoxical. To them there is only one (up to unitary equivalence) "unweighted bilateral shift", the operator $S$ defined by

$$
S \sum_{-\infty}^{\infty} b_{n} y_{n}=\sum_{-\infty}^{\infty} b_{n} y_{n+1}
$$

where $\left\{y_{n}\right\}_{-\infty}^{\infty}$ is an orthonormal basis in Hilbert space and the $b_{n}$ are scalars. That operator is unitary. To get the example of our title, (1) will instead define an operator on Banach space while $\left\{y_{n}\right\}_{-\infty}^{\infty}$ will be a Schauder basis with $\left\|y_{n}\right\|=1$ for all $n$ (the unweightedness).

Given a space $B$ with Schauder basis $\left\{y_{n}\right\}_{-\infty}^{\infty}$, let us interpret (1) as defining a linear operator $S$ with domain precisely that linear manifold of $B$ on which the formula makes sense. The operator $S$ is defined by a matrix with respect to a Schauder basis and hence $[4,11.3$, Corollary 5] is closed. Also, it is clear that the domain of $S$ is dense. Hence the closed graph theorem may be applied to yield the following

CRITERION. $S$ is bounded iff for each element $\sum_{-\infty}^{\infty} b_{n} y_{n}$ in $B, \Sigma_{-\infty}^{\infty} b_{n} y_{n+1}$ also is an element of $B$. Equivalently, $S$ is unbounded iff there is an element $\sum_{-\infty}^{\infty} b_{n} y_{n}$ of $B$ such that $\sum_{-\infty}^{\infty} b_{n} y_{n+1}$ does not converge to an element of $B$.

$S$ is $1-1$ and hence has a left inverse $S^{-1}$ defined by

$$
S^{-1} \sum_{-\infty}^{\infty} b_{n} y_{n}=\sum_{-\infty}^{\infty} b_{n} y_{n-1} \text {. }
$$

An analogous criterion can determine the boundedness of $S^{-1}$.

The space of our example was invented by Nakano.

THEOREM. (See [2] and [3, \$89] for the proofs of the nontrivial parts of this theorem.) Given a bounded sequence $\left\{p_{n}\right\}_{-\infty}^{\infty}$ of real numbers $\geqslant 1$, the collection $B$ of complex sequences $\left\{b_{n}\right\}_{-\infty}^{\infty}$, such that $\sum_{-\infty}^{\infty}\left|b_{n}\right|^{p_{n}}<\infty$, forms a Banach space under the usual algebraic operations and the norm $\left\|\left\{b_{n}\right\}_{-\infty}^{\infty}\right\|=N$, where $N$ is the unique nonnegative real such that $\sum_{-\infty}^{\infty}\left|b_{n} / N\right|^{p_{n}}=1$. If $y_{n}$ is the sequence with 1 in the nth place and 0 in the other places then $\left\{y_{n}\right\}_{-\infty}^{\infty}$ is a Schauder basis in $B .\left\{y_{n}\right\}_{-\infty}^{\infty}$ is an absolute basis (i.e. if $\Sigma b_{n} y_{n} \in B$ and Received by the editors February 19, 1976.

AMS (MOS) subject classifications (1970). Primary 47B99, 47A10.

Key words and phrases. Shift operator, weighted shift operator, sequence space.

(c) American Mathematical Society 1977 
$\left|c_{n}\right| \leqslant M$, all $n$, then $\sum c_{n} b_{n} y_{n} \in B$ ).

EXAMPLE. Let $B$ be defined as in the above theorem with

$$
\begin{aligned}
& p_{n}=1 \quad \text { for } n \leqslant 1, \\
& p_{n}=2\left(\left(2^{k}-1\right) / n\right) \text { for } n \geqslant 2, \text { where } 2^{k}-1 \leqslant n \leqslant 2^{k+1}-2 .
\end{aligned}
$$

Then (a) $S$ is bounded but (b) $S^{-1}$ is unbounded.

Proof. Let $\alpha_{n}=p_{n} / p_{n-1}$. For $n \leqslant 2, \alpha_{n}=1$. For $n>2, \alpha_{n}=(n-1) / n$ $=1-(1 / n)$ unless $n=2^{k}-1$ in which case $p_{n}=2, p_{n-1}=1$ and $\alpha_{n}=2$.

(a) Apply the criterion. Suppose $\left\{b_{n}\right\}_{-\infty}^{\infty} \in B$. That is $\sum v_{n}<\infty$ where $v_{n+1}=\left|b_{n}\right|^{p_{n}}$. It must be shown that $\sum\left|b_{n-1}\right|^{p_{n}}<\infty$. But

$$
\begin{aligned}
\sum\left|b_{n-1}\right|^{p_{n}} & =\sum\left(\left|b_{n-1}\right|^{p_{n-1}}\right)^{\left(p_{n} / p_{n-1}\right)}=\sum v_{n}^{\alpha_{n}} \\
& \leqslant \sum_{-\infty}^{2} v_{n}+\sum_{3}^{\infty} v_{n}^{2}+\sum_{3}^{\infty} v_{n}^{1-(1 / n)} .
\end{aligned}
$$

The convergence of $\sum v_{n}$ implies the convergence of each of the last three sums; only the third sum needs checking. If $v_{n} \leqslant e^{-n}$ then $v_{n}^{1-(1 / n)} \leqslant$ $e^{-n(1-(1 / n))}=e^{-n+1}$. If $v_{n} \geqslant e^{-n}$ then $v_{n}^{1 / n} \geqslant e^{-1}$, so $v_{n}^{1-(1 / n)} \leqslant e v_{n}$. Thus the third sum converges too.

(b) We must find a sequence $\left\{b_{n}\right\}_{-\infty}^{\infty}$ such that $\Sigma\left|b_{n}\right|^{p_{n}}<\infty$ and $\Sigma\left|b_{n}\right|^{p_{n-1}}$ $=\infty$. But $\Sigma\left|b_{n}\right|^{p_{n-1}}=\Sigma\left(\left|b_{n}\right|^{\left.p_{n}\right)^{\alpha_{n}^{-1}}}\right.$ and since $\alpha_{n}^{-1}=\frac{1}{2}$ for an infinite number of $n$ there is no difficulty in finding such a sequence.

Comment. It was conjectured in [1] that the weighted shift in Banach space

$$
S_{\mathrm{a}} \sum_{-\infty}^{\infty} b_{n} y_{n}=\sum_{-\infty}^{\infty} a_{n+1} b_{n} y_{n+1}
$$

(for a fixed sequence of never-zero weights $\mathbf{a}=\left\{a_{n}\right\}_{-\infty}^{\infty}$ ) has its spectrum determined by the sequence a alone, regardless of the space or the basis. The precise conjecture was that if $S_{\mathrm{a}}$ is bounded, spectrum $S_{\mathrm{a}}$ is the closed zero-centered annulus with radii

$$
\lim _{n \rightarrow \infty}\left(\sup _{m} \prod_{i=m+1}^{m+n}\left|a_{i}\right|\right)^{1 / n} \text { and } \lim _{n \rightarrow \infty}\left(\inf _{m} \prod_{i=m+1}^{m+n}\left|a_{i}\right|\right)^{1 / n} .
$$

According to this formula, spectrum $S$ should be the unit circle. However, it can be shown that spectrum $S$ for the above example is a zero-centered disc. Can an example be constructed for which spectrum $S$ is not symmetric about the origin?

\section{REFERENCES}

1. Ralph Gellar, Operators commuting with a weighted shift. Proc. Amer. Math. Soc. 23 (1969), 538-545. MR 41 \#4277a.

2. Hidegorô Nakano, Modulared sequence spaces, Proc. Japan Acad. 27 (1951), 508-512. MR 13, 954.

3. __ Topology and linear topological spaces, Maruzen, Tokyo, 1951. MR 13, 753.

4. Albert Wilansky, Functional analysis, Blaisdell, New York and London, 1964. MR 30 $\# 425$.

Department of Mathematics, North Carolina State University, Ralfigh, North CAROLINA 27607 